\title{
Slow and frozen light in optical waveguides with multiple gratings: Degenerate band edges and stationary inflection points
}

\author{
Nadav Gutman* and C. Martijn de Sterke \\ IPOS and CUDOS, School of Physics, University of Sydney, NSW 2006, Australia \\ Andrey A. Sukhorukov \\ Nonlinear Physics Centre and CUDOS, Research School of Physics and Engineering, Australian National University, \\ Canberra, ACT 0200, Australia \\ Lindsay C. Botten \\ CUDOS, School of Mathematical Sciences, University of Technology, Sydney, NSW 2007, Australia
}

(Received 5 January 2012; published 7 March 2012)

\begin{abstract}
We show that a waveguide with multiple gratings can have a modal dispersion relation which supports frozen light. This means that light can be coupled efficiently to low group velocity modes of an optical waveguide or can even have finite coupling to zero group velocity modes. These effects are associated with stationary points in the dispersion of the form $\omega-\omega_{o} \propto\left(k-k_{o}\right)^{m}$, for integer order $m>1$, around a center frequency $\omega_{o}$ and wave number $k_{o}$. Stationary points of any order can be created, not only regular band edges $(m=2)$, but also degenerate band edges ( $m>2$ and even) and stationary inflection points ( $m$ odd). Using the perturbation theory of matrices in Jordan normal form, the modes and their properties are calculated analytically. Efficient coupling is shown to stem from evanescent modes which must accompany the presence of high-order stationary points with $m>2$.
\end{abstract}

DOI: 10.1103/PhysRevA.85.033804

PACS number(s): 42.70.Qs, 42.81.-i, 41.20.Jb, 78.20.Bh

\section{INTRODUCTION}

The ability to engineer the dispersion of light has been pursued for many years. In the last decade, photonic crystals (PCs), including Bragg gratings, have been proposed and used to control the propagation of light by creating stop bands and by adjusting its group velocity $v_{g}$. There is particular interest in this structural slow light [1-4], in which the light slows down because of the structural properties of the medium, since the field amplitude increases proportionally to the group index $n_{g}=c / v_{g}$ [5]. This enhances linear processes such as absorption or scattering [6,7], since the absorbed and scattered energy scales with the field density. Cubically nonlinear effects such as self-phase modulation, third-harmonic generation, and four-wave mixing are strongly enhanced, since these effects increase quadratically with $n_{g}[8,9]$.

The notion of low and zero group velocities derives from the van Hove singularities [10], where the relation between the frequency of light $\omega$ and its wave vector $k$ is, to lowest order, quadratic, i.e., $\left(\omega-\omega_{o}\right) \propto\left(k-k_{o}\right)^{2}$, where $\omega_{o}$ and $k_{o}$ are the center frequency and wave number, respectively. The associated group velocity, corresponding to the first derivative $v_{g}=\partial \omega / \partial k$, can be small or even zero. When considering simple periodic optical structures such as one-dimensional (1D) dielectric PCs or fibers with a single Bragg grating, such quadratic stationary points (SPs) occur in the center and at the edge of the Brillouin zone (BZ). In more complex structures, such as two- and three-dimensional (2D, 3D) PCs or photonic crystal waveguides (PCWs), quadratic SPs can appear at other positions in the BZ and at high-symmetry points [11]. In 1D structures an SP can occur at any position in the BZ if the

\footnotetext{
*nadav@physics.usyd.edu.au
}

structure is made of nonreciprocal material [2] or if different gratings are superimposed in optical fiber [3].

SPs can be of higher order than quadratic, for which the dispersion relation can be written as

$$
\left(\omega-\omega_{o}\right) \propto\left(k-k_{o}\right)^{m}
$$

for any integer $m>1$. Three cases need to be differentiated: regular band edges (RBEs) for which $m=2$; degenerate band edges (DBEs) for even $m$ and $m>2$; and stationary inflection points (SIPs) for odd $m$ 's. As mentioned, RBEs occur in any periodic structure; in contrast, DBE and SIP need to be specifically engineered.

The first theoretical investigation of general SPs in PCs was reported by Figotin and Vitebskiy [2]. They showed that cubic SIPs $\left(k^{3}\right)$ and a quartic DBE $\left(k^{4}\right)$ can be generated in a 1D magnetic PC. In 1D PCs, birefringence materials [2] or tilted incident angles $[12,13]$ with nonreciprocal materials are required to create high-order SPs, and the maximum achievable reported by these authors was $m=4$.

Slow light, including that close to an SP, is most effective in guided-wave structures where transverse light confinement enhances the field strength (in addition to the enhancement from slow-light effects). Slow light in PCW was successfully demonstrated, both for RBE [14] and more recently near a cubic SIP [15]. A quartic DBE was found both theoretically [16] and experimentally [17] in coupled periodic waveguides.

Unlike the 1D PCs considered by Figotin et al. [2,18,19], where at any frequency there are four modes, in optical waveguides the number of modes may be chosen by varying the waveguide's cross section. In this paper we focus on these geometries, and our aims are threefold. First, following preliminary work [20], we show that both SIPs and DBEs with arbitrary $m$ can be created in a guided structure by coupling between the various modes using multiple gratings, 


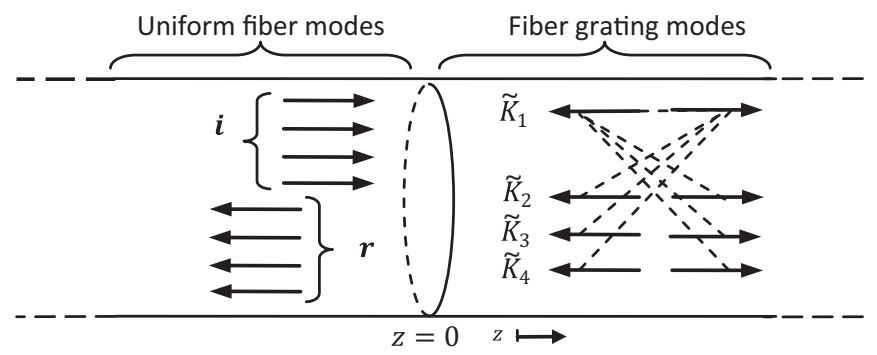

FIG. 1. Schematic of our approach to generate SPs. For $z<0$ the multimode waveguide has $s$ incident and $s$ reflected modes. For $z>0$ there are multiple gratings with different periods, coupling a single forward mode to all backward modes and vice versa

as illustrated Fig. 1. The second aim is to analyze the wave propagation for frequencies around SIPs and DBEs by using coupled mode theory [21]. Using this theory, which applies to shallow gratings, the entire problem can be analyzed using the linear algebra of defective matrices. This approach complements that of Figotin et al., who use a transfer matrix approach. Third, even though the theory applies strictly speaking to shallow gratings, many of our findings are generic and apply to any SIP or DBE, for example, SIP in PCWs [22]. Although these have been studied numerically and experimentally [23], the complexity of these structures did not allow for a comprehensive theoretical analysis.

The most significant difference between RBE and highorder SPs is the existence of degenerate evanescent modes in addition to the propagating modes [24]. Briefly, these evanescent modes play a significant role at the interfaces of structures with SPs, as they help fulfill the boundary condition. At the interface between a fast and a slow medium, for example, the evanescent mode(s) and the slowly propagating mode have large and opposite amplitudes, allowing for efficient coupling to a fast mode. Since the evanescent mode(s) decay away from the interface, only the large-amplitude slowly propagating mode remains. This is illustrated in Fig. 2. We investigate the evanescent modes of SPs and show analytically how their presence affects the coupling efficiency.

The paper is organized as follows: In Sec. II we formulate a general approach to calculate the dispersion of a waveguide with multiple gratings. Matrices in the Jordan normal forms are used to describe SPs and the connection between the number of modes and the achievable orders of SP. Perturbation theory of such matrices is used to determine the dispersion and the associated waveguide modes, close to a SP. In Sec. III, the coupling problem to a waveguide with SPs in its dispersion is solved for DBEs and SIPs. The relationship between the number of evanescent modes and the coupling efficiency is shown. In Sec. IV we discuss and conclude our findings and point out the difference between SIP and DBE with respect to the mode structure.

\section{OPTICAL WAVEGUIDES WITH MULTIPLE BRAGG GRATINGS}

We consider an optical waveguide which supports $s$ optical modes at a frequency $\tilde{\Omega}$. The $s$ optical modes differ from
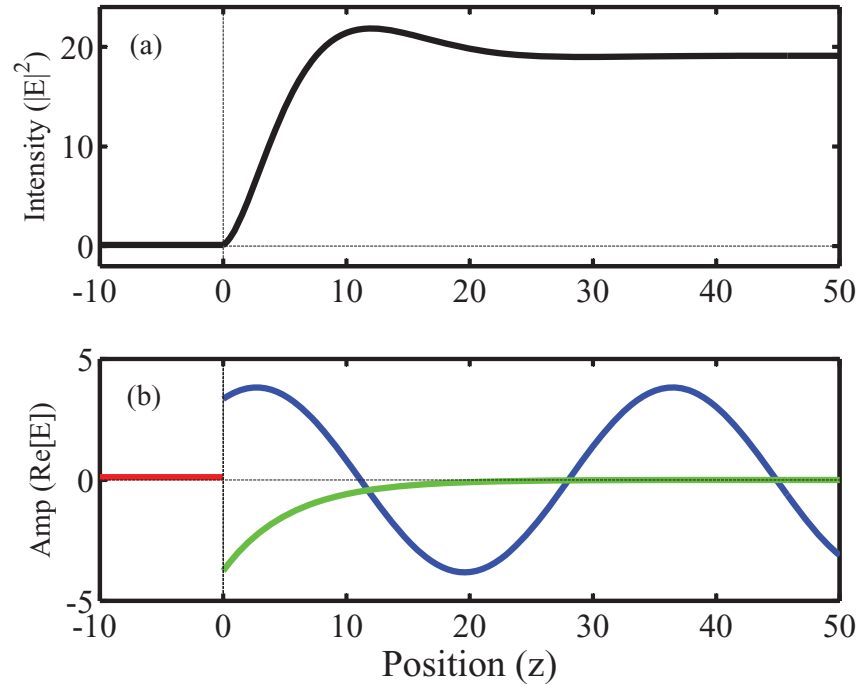

FIG. 2. (Color online) (a) The total field and (b) the amplitudes of the propagating and evanescent waves on both sides of the interface. (b) Shows how the boundary condition between a fast incident mode (left red) and a slow, high-amplitude mode (blue-oscillating) can be facilitated by an evanescent mode (green-decaying). The slow and evanescent modes have large amplitudes; at the interface they interfere destructively, so the resulting low-amplitude field matches the incoming mode. Far from the interface the total field (black) consists of only the slow mode. (a) The square sum of the fields in (b).

each other by their wave numbers $\tilde{K}_{j}$, where $j=1, \ldots, s$. To manipulate the dispersion of the light inside the waveguide and to create SPs, as in Eq. (1), a set of gratings with different periods, amplitudes, and transverse profiles is superimposed in some part of the waveguide. To have the largest number of degrees of freedom, $s$ gratings are used. As shown below, this enables us to generate an SP with a maximum even order of $m=2 s$ and a maximum odd order of $m=s$.

Each waveguide mode $j$ can travel forward or backward with propagation constants $\pm \tilde{K}_{j}$, respectively. Gratings can couple two different forward or two different backwardpropagating modes $p$ and $q$; such long period gratings have a period $\Lambda$ satisfying $2 \pi / \Lambda=\left|\tilde{K}_{p}-\tilde{K}_{q}\right|$. Gratings can also couple a forward-propagating mode to a backward one; such Bragg gratings need to have a much smaller period $2 \pi / \Lambda=$ $\left|\tilde{K}_{p}+\tilde{K}_{q}\right|$. Though long-period gratings and Bragg gratings appear to have the same coupling properties [3], in this paper we only consider Bragg gratings. This choice is related to the need to avoid coupling to radiative modes, as discussed below.

The Bragg gratings are used to couple all forwardpropagating modes to a single backward mode (mode 1), and so they also couple all backward modes to the forward mode 1 (see Fig. 1). The Bragg gratings have a period of $\Lambda_{j}$, $2 \pi / \Lambda_{j}=\tilde{K}_{1}+\tilde{K}_{j}+\delta_{j}$, where $\delta_{j}$ are small detunings from the respective Bragg conditions. A common way to create gratings in optical fibers is by exploiting the photorefractivity of glass by which the material absorbs UV radiation and changes its refractive index [25]. A refractive index difference up to $10^{-3}$ is achievable in silica glass [26]. Superimposing $s$ dielectric gratings thus induces a dielectric constant 
modulation of

$$
\Delta \epsilon(x, y, z)=\sum_{j=1}^{s} \Delta \epsilon_{j} \cos \left[\left(\kappa_{j}+\delta_{j}\right) z\right] R_{j}(x, y),
$$

where $z$ is the propagation direction, and $\Delta \epsilon_{j}$ and $R_{j}(x, y)$ are, respectively, the strength and the transverse profile of grating $j$. When coupling between modes of the same symmetry, e.g. $\mathrm{LP}_{01}$ and $\mathrm{LP}_{02}$ of an optical fiber, then $R_{j}(x, y)$ cannot be antisymmetric since otherwise the coupling strength vanishes. Similarly, for modes with different symmetry, e.g., $\mathrm{LP}_{01}$ and $\mathrm{LP}_{11}, R_{j}(x, y)$ cannot be symmetric.

To find the dispersion of the perturbed system, we assume that the structure is much longer than the beat length between any two modes, or $L \gg\left|\tilde{K}_{1}+\tilde{K}_{j}\right|^{-1},\left|\tilde{K}_{1}\right|^{-1},\left|\tilde{K}_{j}\right|^{-1}$, which is satisfied under typical conditions. To solve the Maxwell's equations we write the total field as a superposition of all modes of the uniform waveguide, each with an envelope dependent on $z$ and $t$. Therefore,

$$
\begin{aligned}
E(x, y, z, t)= & \sum_{j=1}^{s} g_{j}(x, y)\left[E_{j}^{+}(z, t) e^{i \tilde{K}_{j} z}\right. \\
& \left.+E_{j}^{-}(z, t) e^{-i \tilde{K}_{j} z}\right] e^{-i \tilde{\Omega} t}+\text { c.c. },
\end{aligned}
$$

where $E_{j}^{ \pm}(z, t)$ are the forward- and backward-propagating envelopes of mode $j$, the $g_{j}(x, y)$ are the transverse mode profiles, and c.c. denotes the complex conjugate. Substituting ansatz (3) into the wave equation leads to a set of $2 s$ equations, one each at spatial frequency $\exp \left( \pm \tilde{K}_{j} z\right)$. The other spatial frequencies, $\pm 3 \tilde{K}_{1}, \pm 2 \tilde{K}_{1} \mp \tilde{K}_{j}, \pm 2 \tilde{K}_{1} \pm \tilde{K}_{j}, \pm 2 \tilde{K}_{j} \pm \tilde{K}_{1}$, $\pm \tilde{K}_{1} \pm \tilde{K}_{j} \pm \tilde{K}_{l}$, and $\pm \tilde{K}_{1} \pm \tilde{K}_{j} \mp \tilde{K}_{l}$, where $j, l \neq 1$, are not phase matched to the envelopes of $E_{j}^{ \pm}$and their contribution is negligible for shallow gratings [3].

Radiative modes have wave numbers above the light line. To prevent the grating from coupling light into radiative modes, the spatial frequencies listed above must not match the wave numbers of radiative modes. Hence, they must be at least as large as the wave number of the smallest guided modes: $\min \left\{\tilde{K}_{j}\right\}$. It is sufficient to require that the smallest spatial frequencies are larger than this mode. Thus we require

$$
\left|\tilde{K}_{1}+\tilde{K}_{j}-\tilde{K}_{l}\right|, \quad\left|2 \tilde{K}_{1}-\tilde{K}_{j}\right|>\min \left\{\tilde{K}_{j}\right\} .
$$

To satisfy inequality (4), $\tilde{K}_{1}$ can be chosen to be the largest. This requirement can be fulfilled more easily with Bragg gratings than with long period gratings.

The key idea behind time-dependent coupled mode theory (CMT) [21] is that the $\Delta \epsilon_{j}$ are small, so the $E_{j}^{ \pm}$change slowly with $z$ and $t$. A set of $2 s$ equations is obtained using CMT, each of which corresponds to a field with a wave number $\pm \tilde{K}_{j}$ at the center frequency $\tilde{\Omega}$. Each equation is multiplied by the $g_{j}(x, y)$ and integrated over both $x$ and $y$. This defines the parameters

$$
\rho_{j}=\frac{\Delta \epsilon_{j} \tilde{\Omega}}{2 c} \int_{-\infty}^{\infty} g_{1}(x, y) R_{j}(x, y) g_{j}(x, y) d x d y,
$$

which are the coupling coefficients between the modes 1 and $j$. Near $\tilde{\Omega}$, the unperturbed waveguide dispersion is, to lowest order, $(\Omega-\tilde{\Omega})=v_{j}\left(K-\tilde{K}_{j}\right)$, where the $v_{j}$ are the group velocities of the modes. The final set of equations is [3]

$$
\begin{aligned}
i \frac{\partial E_{1}^{ \pm}}{\partial t} \pm i v_{1} \frac{\partial E_{1}^{ \pm}}{\partial z}+\sum_{j=1}^{s} \rho_{j} E_{j}^{\mp} e^{ \pm i \delta_{j} z} & =0, \\
i \frac{\partial E_{j}^{ \pm}}{\partial t} \pm i v_{j} \frac{\partial E_{j}^{ \pm}}{\partial z}+\rho_{j} E_{1}^{\mp} e^{ \pm i \delta_{j} z} & =0 .
\end{aligned}
$$

To find the dispersion relation we take plane-wave solutions for the $E_{j}^{ \pm}$, so

$$
\begin{aligned}
& E_{1}^{ \pm}=A_{1}^{ \pm} e^{i\left(k \pm \delta_{1} / 2\right) z-i \omega t}, \\
& E_{j}^{ \pm}=A_{j}^{ \pm} e^{i\left(k \mp \delta_{1} / 2 \pm \delta_{j}\right) z-i \omega t},
\end{aligned}
$$

where the $A_{j}$ 's are constants and $\omega$ and $k$ are the frequency and wave-number difference from $\tilde{\Omega}$ and $\tilde{K}_{j}$, respectively. By substituting Eqs. (7) into (6), we obtain a set of linear algebraic equations which can be represented as a $2 s \times 2 s$ matrix $\Theta$, termed the propagation matrix. This constitutes an eigenvalue problem in $k$. Assuming a given frequency $\omega$, the $k$ 's are the eigenvalues of

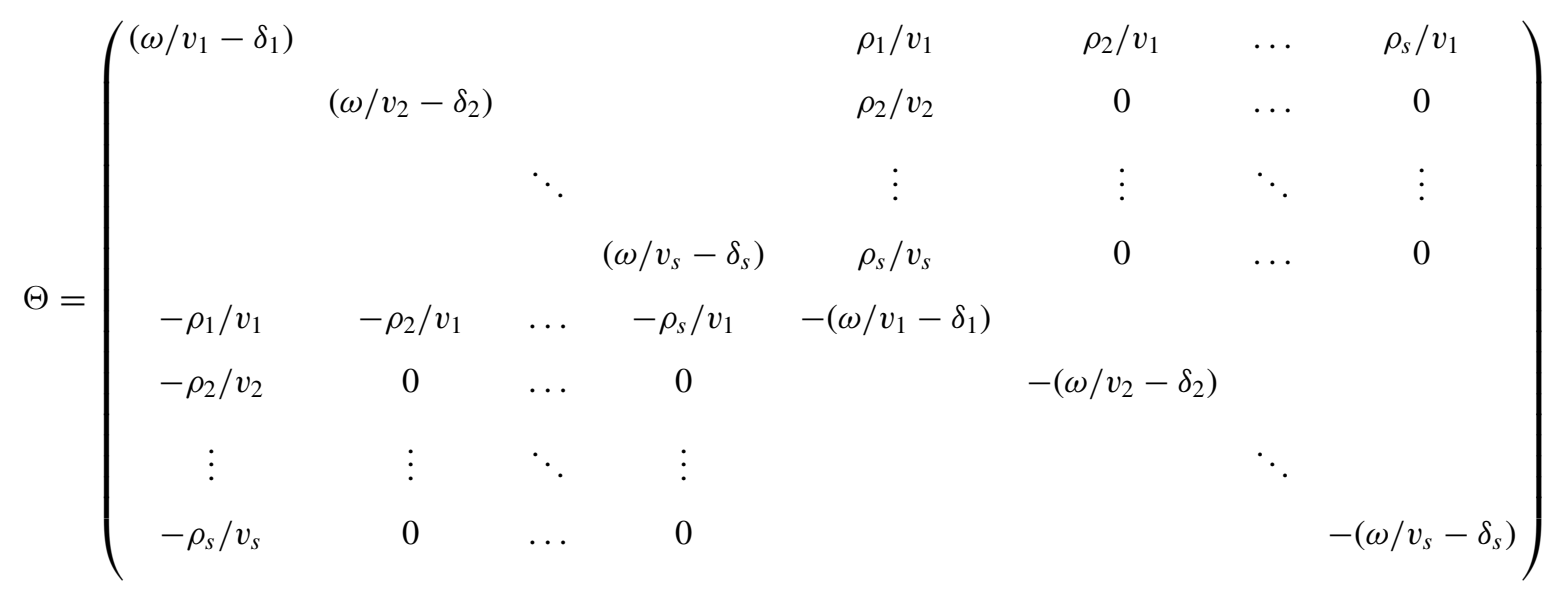


(a)

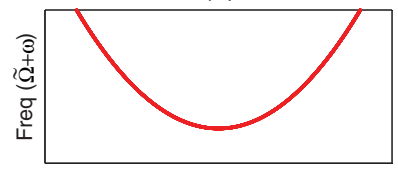

(c)

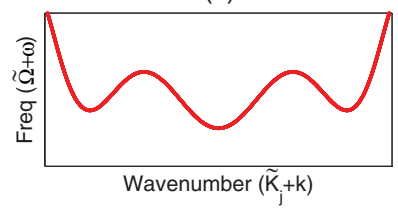

(b)

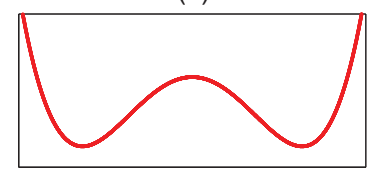

(d)

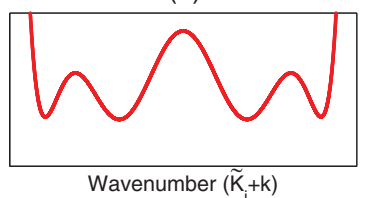

FIG. 3. (Color online) Typical dispersion curves showing frequency versus (real) wave number around $\tilde{\Omega}$ and $\tilde{K}_{j}$ for (a) one, (b) two, (c) three, and (b) four modes and gratings.

In the corresponding eigenvectors the first $s$ elements are the amplitudes of the forward-propagating modes, whereas the remaining $s$ elements are the amplitudes of the backwardpropagating modes, i.e.,

$$
\Theta \mathbf{f}^{(k)}=k\left(\begin{array}{c}
\mathbf{f}^{+(k)} \\
\mathbf{f}^{-(k)}
\end{array}\right)=k\left(\begin{array}{c}
A_{1}^{+} \\
\vdots \\
A_{s}^{+} \\
A_{1}^{-} \\
\vdots \\
A_{s}^{-}
\end{array}\right) .
$$

For most sets of parameters $\left(v_{j}, \rho_{j}, \delta_{j}\right)$ the matrix $\Theta$ can be diagonalized, where the diagonal entries are the eigenvalues, corresponding to the wave numbers. Figure 3 shows the detuned frequency $\omega$ from the center frequency $\tilde{\Omega}$ versus the real solutions to the wave number $\tilde{K}_{j}+k$. Solutions with real $k$ 's ("grating modes") represent propagating modes in the grating. These modes are not Bloch modes since they have more than one periodicity in the direction of the waveguide. If for a given frequency the number of solutions with real wave numbers is less than $2 s$, the other solution must be complex. Since $\Theta$ is real, the $k$ 's then come in complex conjugate pairs. As the waveguide is made of reciprocal material, if $k$ is a solution, then so is $-k$. Therefore, in the most general case the eigenvalues come in groups of four, namely,

$$
k,-k, k^{\star},-k^{\star} \text {. }
$$

For parameters where $\Theta$ cannot be diagonalized and hence is defective, it can be brought to a Jordan normal from. In the following section we show that such parameters are associated with an SP.

\section{A. Stationary points and the Jordan normal form of propagation matrix $\Theta$}

A matrix can always be brought to a Jordan normal form $J$, where $\Theta=S J S^{-1}$. If the matrix is defective it consist of a Jordan block of size $m>1$. In this section we show that the size of the Jordan block $m$ is associated with an SP of order

$m$. The Jordan blocks corresponding to a quadratic $\left(k^{2}\right)$, cubic $\left(k^{3}\right)$, and quartic $\left(k^{4}\right)$ are, respectively,

$$
J_{m}=\left(\begin{array}{cc}
k_{o} & 1 \\
& k_{o}
\end{array}\right),\left(\begin{array}{ccc}
k_{o} & 1 & \\
& k_{o} & 1 \\
& & k_{o}
\end{array}\right),\left(\begin{array}{cccc}
k_{o} & 1 & & \\
& k_{o} & 1 & \\
& & k_{o} & 1 \\
& & & k_{o}
\end{array}\right),
$$

where the other elements are zero.

A Jordan block is a defective matrix, which has only a single eigenvector and eigenvalue. This is true when two or more eigenvalues become degenerate, say at frequency $\omega_{o}$. We now consider how the eigenvalues and eigenvectors approach each other as $\Delta \omega \equiv \omega-\omega_{o} \rightarrow 0$, which determines the order of the SP. For simplicity we consider only a Jordan block of size $m$ and not a complete matrix $\Theta$. At a frequency detuned from the defective matrix condition by $\Delta \omega$ the perturbed problem is

$$
\left(J_{m}+\Delta \omega B\right) \mathbf{V}^{(k)}=\left(k_{o}+\Delta k\right) \mathbf{V}^{(k)},
$$

where $\mathbf{V}^{(k)}$ is the eigenvector in the Jordan normal base and $B$ is a matrix.

According to Lidskii's perturbation theory of matrices in the Jordan normal form $[27,28]$, the perturbed eigenvalues can be written as

$$
\Delta k=\sqrt[m]{\xi \Delta \omega}
$$

where $\xi$ is the bottom left element of matrix $B\left(\xi=B_{m, 1}\right)$. In brief, this can be shown by expanding $\left(J_{m}+\Delta \omega B\right)^{m} \mathbf{V}^{(k)}=$ $\left(k_{o}+\Delta k\right)^{m} \mathbf{V}^{(k)}$ and taking the leading order in $\Delta \omega$. Equation (13) describes the relationship between the size of the Jordan block and the associated order $m$ of the SP, since $\Delta \omega \propto \Delta k^{m}$.

Equation (13) not only gives the real wave numbers close to the SP, but also the complex and imaginary ones. It shows that the roots of $\Delta \omega$ consist of $|\Delta k|$ multiplied by all the $m$ roots of unity $\sqrt[m]{1}$. For positive $\xi$,

$$
\Delta k_{j} / \sqrt[m]{\xi \Delta \omega}=\left\{\begin{array}{ll}
\chi^{j} & \Delta \omega>0 \\
\chi^{j} e^{i \pi / m} & \Delta \omega<0
\end{array},\right.
$$

where $j=0,1, \ldots,(m-1)$ and $\chi=\exp (i 2 \pi / m)$. In Fig. 4 the dispersion relation close to a quadratic $\left(k^{2}\right)$, cubic $\left(k^{3}\right)$, quartic $\left(k^{4}\right)$, quintic $\left(k^{5}\right)$, sextic $\left(k^{6}\right)$, and octic $\left(k^{8}\right)$ SPs are illustrated.

To transform matrix $\Theta$ to the Jordan normal form, the basis of each Jordan block is generated from the block's single eigenvector $\mathbf{f}_{1}^{\left(k_{o}\right)}$. Following the Jordan chain process [29], the other vectors are found by

$$
\Theta^{j-1} \mathbf{f}_{j}^{\left(k_{o}\right)}=\mathbf{f}_{1}^{\left(k_{o}\right)}
$$

for $j=1, \ldots,(m-1)$. The transformation matrix from the waveguide modes base $\mathbf{f}_{j}^{\left(k_{o}\right)}$ to the Jordan base is

$$
S_{m}=\left[\begin{array}{ll}
\mathbf{f}_{1}^{\left(k_{o}\right)} & \mathbf{f}_{2}^{\left(k_{o}\right)} \ldots \mathbf{f}_{m}^{\left(k_{o}\right)}
\end{array}\right]
$$


(a)
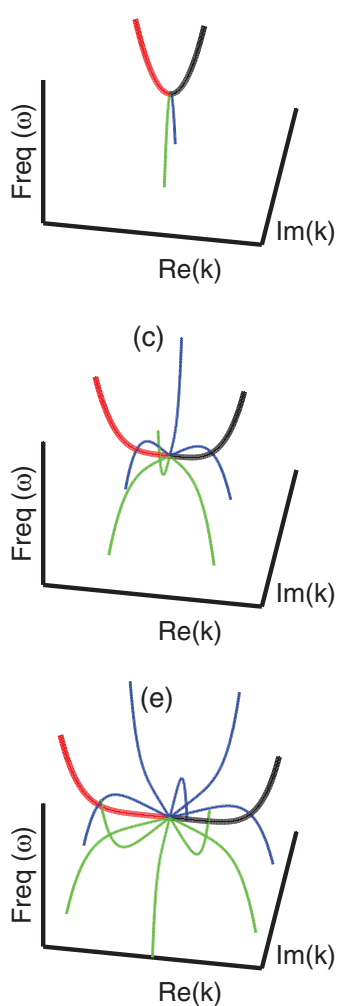

(b)
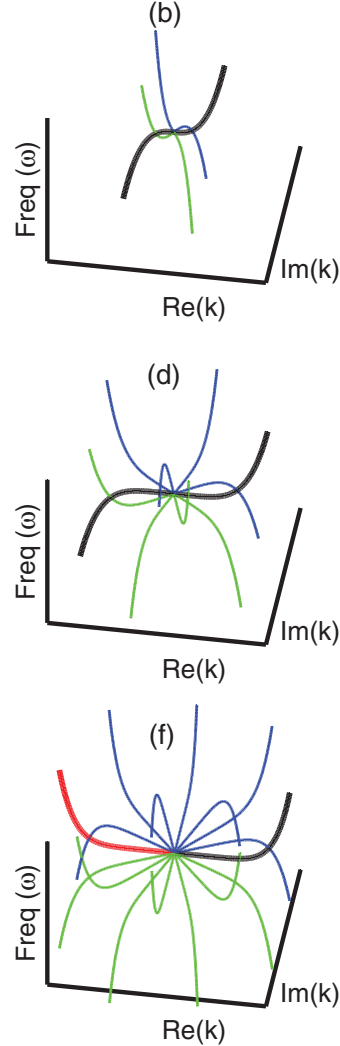

FIG. 4. (Color online) Complex dispersion relation around SPs, showing frequency versus the real and imaginary parts of the wave number. (a) Quadratic, (b) cubic, (c) quartic, (d) quintic, (e) sextic, and (f) octic. Black and red (gray) thick lines represent forward- and backward-propagating modes, respectively. Blue (gray) and green (light gray) thin lines represent forward- and backward-decaying evanescent modes.

At the SP frequency, $\Theta\left(\omega_{o}\right)=J_{m}$. For a frequency detuned from this point, $\Theta$ is expressed as $\Theta=S_{m}\left[J_{m}+(\Delta \omega) B\right] S_{m}^{-1}$, where

$$
B=S_{m}^{-1}\left(\begin{array}{cccccc}
1 / v_{1} & & & & & \\
& \ddots & & & & \\
& & 1 / v_{s} & & & \\
& & & -1 / v_{1} & & \\
& & & \ddots & \\
& & & & -1 / v_{s}
\end{array}\right) S_{m} .
$$

Using $B$, the parameter $\xi$, which completely determines the dispersion, is calculated.

The perturbation theory of the Jordan normal form also shows that the eigenvector can be described to lowest order as

$$
\mathbf{V}^{\left(k_{j}\right)}=\left[\begin{array}{ll}
1 & \Delta k_{j} \ldots \Delta k_{j}^{m-1}
\end{array}\right]^{T}
$$

where $T$ stands for the transpose, associated with the wave number $k_{o}+\Delta k_{j}$. Using this, the set of grating modes can be described more simply by using the Vandermonde matrix [29]

$$
\begin{aligned}
\mathcal{F}_{m}= & S_{m}\left(\begin{array}{cccc}
1 & & & \\
& \Delta k & & \\
& & \ddots & \\
& & & \Delta k^{m-1}
\end{array}\right) \\
& \times\left(\begin{array}{cccc}
1 & 1 & & 1 \\
1 & \chi & & \chi^{m-1} \\
\vdots & \vdots & \ddots & \vdots \\
1 & \chi^{m-1} & & \chi^{(m-1)(m-1)}
\end{array}\right),
\end{aligned}
$$

where the right-hand matrix is the Vandermonde matrix. Each column of the matrix $\mathcal{F}_{m}$ is an eigenmode in the grating waveguide ( $z>0$ in Fig. 1), where the column entries are the amplitudes of the homogeneous waveguide modes $(z<0$ in Fig. 1).

\section{B. Condition for a stationary point}

Here we formalize the condition on the matrix $\Theta$ to have a Jordan block of size $m$. The characteristic polynomial of the matrix $\Theta$ is

$$
p(k)=|\Theta-k I| .
$$

The characteristic polynomial of a Jordan block of size $m$ is $\left|J_{m}-k_{o} I_{m}\right|=\left(k-k_{o}\right)^{m}$. Since the characteristic polynomial of the entire matrix $p(k)$ is the multiplication of the characteristic polynomial of each of its blocks, either diagonalized or not, $p(k)$ can be divided by $\left(k-k_{o}\right)^{m}$. Thus all the first $m-1$ derivatives of $p(k)$ at $k_{o}$ must be equal to zero. This always holds provided that $\Theta$ is nonderogatory, which means that it does not have two or more equal eigenvalues that do not belong to the same Jordan block. Since $k_{o}$ also needs to be a root of the characteristic polynomial, the total $m$ conditions for a Jordan block of size $m$ are

$$
\left.\frac{\partial^{j} p(k)}{\partial k^{j}}\right|_{k=k_{o}}=0,
$$

where $j=0, \ldots, m-1$.

\section{Physical preconditions on the size of Jordan blocks}

To preserve the symmetry of the wave number spectrum of $\Theta$, following Eq. (13), Jordan blocks with nonzero eigenvalues must come in pairs with eigenvalues $\pm k_{o}$. When $k_{o}=0$, a single Jordan block of even dimension can exist. However, this limits the largest odd-sized Jordan block to $s$, for a $2 s$ system. The spectrum of matrix $\Theta$ is varied by changing the $\delta_{j}$ 's, and to engineer the matrix to have Jordan blocks of larger sizes, the $\delta_{j}$ 's can be changed to merge two or more quadratic SPs, as illustrated in Fig. 5. We find that the conditions for a high-order SP can easily be found by fixing $k_{o}$ and finding only $\omega$ and $m-1$ values of $\delta_{j}$. The other $\delta_{j}$ can be set to zero, if the matrix dimension (i.e., the number of gratings) is larger than needed. 

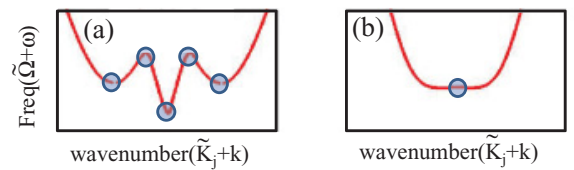

wavenumber $\left(\widetilde{\mathrm{K}}_{\mathrm{j}}+\mathrm{k}\right)$

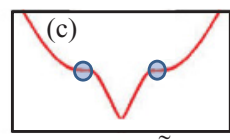

wavenumber $\left(\widetilde{\mathrm{K}}_{\mathrm{j}}+\mathrm{k}\right)$

FIG. 5. (Color online) Dispersion around $k=0$ of a waveguide with three gratings. The generic dispersion has five RBEs. (a) By changing the $\delta_{j}$ 's all RBEs can be merged to create a sextic DBE. (b) Alternatively, the left and right RBEs are tuned to create a cubic SIP, (c) with $\pm k_{o}$. The Jordan normal forms of (b) and (c) are given in Eqs. (22) and (23), respectively.

To illustrate the procedure we use the particular case of a waveguide with three modes, which is described by a $6 \times 6$ matrix. Such a system and matrix can support both even blocks,

$$
\left(\begin{array}{llllll}
0 & 1 & & & & \\
& 0 & 1 & & & \\
& & 0 & 1 & & \\
& & & 0 & 1 & \\
& & & & 0 & 1 \\
& & & & & 0
\end{array}\right)
$$

and odd Jordan blocks,

$$
\left(\begin{array}{ccc|ccc}
k_{o} & 1 & & & & \\
& k_{o} & 1 & & & \\
& & k_{o} & & & \\
\hline & & -k_{o} & 1 & \\
& & & & -k_{o} & 1 \\
& & & & & -k_{o}
\end{array}\right) .
$$

\section{SEMI-INFINITE STRUCTURE}

The coupling to a semi-infinite structure near an SP of order $m$ can be found by matching the amplitudes of the input channels to the forward-going grating modes (19). These have either a positive Poynting vector,

$$
S_{k}=\sum_{j=1}^{s}\left(\left|A_{j}^{-}\right|^{2}-\left|A_{j}^{-}\right|^{2}\right) v_{j}
$$

or decay in the forward direction $\operatorname{Im}(k)>0$. These modes are marked in Fig. 4 as blue and green, respectively. We consider an incident field $\mathbf{i}$, in which each of the $s$ forward-propagating modes of the unperturbed waveguide has a complex amplitude $\left|i_{j}\right| \exp \left(i \phi_{j}\right)$. The grating modes are excited with amplitudes $a_{j}$, as calculated from the incident field, and the reflection in each channel $r_{j}$ is then also calculated. This can be expressed in matrix form as

$$
\begin{aligned}
& \mathbf{i}=\left(\begin{array}{c}
i_{1} \\
\vdots \\
i_{s}
\end{array}\right)=\sum_{j=1}^{s}\left(\mathbf{f}^{+\left(k_{j}\right)} a_{j}\right)=M_{+} \mathbf{a}, \\
& \mathbf{r}=\left(\begin{array}{c}
r_{1} \\
\vdots \\
r_{s}
\end{array}\right)=\sum_{j=1}^{s}\left(\mathbf{f}^{-\left(k_{j}\right)} a_{j}\right)=M_{-} \mathbf{a} .
\end{aligned}
$$

To find the relation between the incident and the reflection fields, the matrix $R$ is defined as [30]

$$
R=M_{-} M_{+}^{-1} \text {. }
$$

To maintain an incident field with unit intensity, the amplitude in each channel $i_{j}$ is normalized to its group velocity $v_{j}$ as

$$
1=\sum_{j=1}^{s}\left|i_{j}\right|^{2} v_{j}
$$

The total reflection and the associated energy coupling efficiency are then

$$
\eta=1-\sum_{j=1}^{s}\left|r_{j}\right|^{2} v_{j}
$$

Different numbers of modes can be excited by changing the incident vector i. For example, near a quartic SP, for which there is one propagating mode and one evanescent mode [see Fig. 4(c)], both modes are generally excited. For singular initial condition points, either the evanescent or the propagating mode is excited. To calculate the coupling efficiency, it is sufficient to find the amplitude of the propagating mode $a_{\text {prop }}$, since only it carries energy. In Secs. III A and III B we show that the amplitudes of all modes, including the propagating mode, scale as

$$
a_{j} \propto \Delta k^{-l},
$$

where $l$ is the number of degenerate evanescent modes excited simultaneously. The energy flux or the Poynting vector is calculated using $\eta=\left|a_{\text {prop }}\right|^{2} S_{k} \propto\left|a_{\text {prop }}\right|^{2} v_{g}$, where $S_{k}$ is the propagating mode with unit power, found using Eq. (24). Since $v_{g}=m \Delta k^{m-1} / \xi$, the coupling efficiency is

$$
\eta \propto\left|a_{\text {prop }}\right|^{2} v_{g} \propto\left(\frac{1}{\Delta k^{l}}\right)^{2} \Delta k^{m-1}=\Delta k^{m-2 l-1} \propto v_{g}^{\frac{m-2 l-1}{m-1}} .
$$

This is a key result of this paper and shows explicitly how the coupling efficiency scales at different SPs. It shows that the efficiency depends on the number of evanescent modes which are excited and which help in achieving efficient coupling. If all the evanescent modes which are degenerate with the propagating mode are excited, the maximum achievable coupling is $\eta \propto v_{g}^{1 /(m-1)}$ for DBEs. For SIPs which occur for odd $m$, such as cubic $\left(k^{3}\right)$ and quintic $\left(k^{5}\right)$ SIPs, the coupling at zero group velocity scales as $\eta \propto 1$ if all the evanescent modes are excited. This frozen light occurs when energy is coupled to light with zero group velocity [18]. Not all degenerate modes at the SP frequency contribute to enhanced coupling. In general, this occurs where $k_{o} \neq 0$ where more than one Jordan block exists. In Eq. (29) $l$ refers to evanescent modes only from the same Jordan block. Now that Eq. (30) has been established, we prove this result in the remainder of this section for both odd (Sec. III A) and even (Sec. III B) SPs.

We stress that in interpreting these results it is important to recall that the variations of the coupling efficiency to the slow light waveguide depend on the amplitudes and relative phases of the incident light (see left side of Fig. 1). However, as discussed previously [20], the scaling refers to the maximum 
efficiency which can be achieved by optimizing the launch conditions at fixed frequency, and also to efficiency averaged over all these launch conditions.

\section{A. Even stationary points}

Here we prove Eq. (29) for even SPs with $k_{o}=0$, where all forward modes are degenerate. This case is represented by a single Jordan block as in Eq. (22) for a sextic DBEs. Even SPs have a single forward-propagating mode and $s-1$ forward decaying evanescent modes. To solve the amplitudes of each mode $a_{j}$ according to the boundary condition Eq. (25a), we apply Cramer's rule [29],

$$
a_{j}=\frac{\operatorname{det}\left(M_{+}^{(j)}\right)}{\operatorname{det}\left(M_{+}\right)},
$$

where $M_{+}^{(j)}$ is a matrix obtained from $M_{+}$by replacing the $j$ th column with the incident fields $\mathbf{i}$ vector.

\section{Coupling to all the evanescent modes}

To demonstrate our approach, we first consider the case of sextic DBE. Then, the matrix $M_{+}$can be represented using generalized eigenvectors (15) and the eigenvalue (13).

$$
\begin{aligned}
M_{+}= & \left(\begin{array}{c}
\mathbf{f}_{1}^{+\left(k_{o}\right) T}+\Delta k \mathbf{f}_{2}^{+\left(k_{o}\right) T}+\Delta k^{2} \mathbf{f}_{3}^{+\left(k_{o}\right) T} \\
\mathbf{f}_{1}^{+\left(k_{o}\right) T}+\Delta k \chi \mathbf{f}_{2}^{+\left(k_{o}\right) T}+(\Delta k \chi)^{2} \mathbf{f}_{3}^{+\left(k_{o}\right) T} \\
\mathbf{f}_{1}^{+\left(k_{o}\right) T}+\Delta k \chi^{2} \mathbf{f}_{2}^{+\left(k_{o}\right) T}+\left(\Delta k \chi^{2}\right)^{2} \mathbf{f}_{3}^{+\left(k_{o}\right) T}
\end{array}\right)^{T} \\
& +O\left(\Delta k^{3}\right) .
\end{aligned}
$$

More simply, (32) can be represented using a Vandermonde matrix

$$
M_{+}=S_{+}\left(\begin{array}{ccc}
1 & & \\
& \Delta k & \\
& & \Delta k^{2}
\end{array}\right)\left(\begin{array}{ccc}
1 & 1 & 1 \\
1 & \chi & \chi^{2} \\
1 & \chi^{2} & \chi^{4}
\end{array}\right),
$$

where $\chi=e^{i 2 \pi / 6}$, and $S_{+}$is a submatrix of $S$,

$$
S_{+}=\left[\begin{array}{lll}
\mathbf{f}_{1}^{+\left(k_{o}\right)} & \mathbf{f}_{2}^{+\left(k_{o}\right)} & \mathbf{f}_{3}^{+\left(k_{o}\right)}
\end{array}\right] .
$$

From (33) the determinant of $M_{+}$can be easily calculated to be

$$
\operatorname{det}\left(M_{+}\right)=\operatorname{det}\left(S_{+}\right)\left(\Delta k^{3}\right) \operatorname{det}\left(M_{V M}\right),
$$

where $M_{V M}$ is the Vandermonde matrix. This shows that $\operatorname{det}\left(M_{+}\right) \propto \Delta k^{3}$.

Next, we calculate $M_{+}^{(j)}$ by replacing one of the columns of $M_{+}$with the incident field $\mathbf{i}$, which in general is a linear combination of the columns of $S_{+}$, i.e.,

$$
\mathbf{i}=b_{1} \mathbf{f}_{1}^{+\left(k_{o}\right)}+b_{2} \mathbf{f}_{2}^{+\left(k_{o}\right)}+b_{3} \mathbf{f}_{3}^{+\left(k_{o}\right)} \text {. }
$$

To calculate $a_{1}=a_{\text {prop }}$, the propagating mode

$$
\begin{aligned}
M_{+}^{(\text {prop })}= & \left(\begin{array}{c}
b_{1} \mathbf{f}_{1}^{+\left(k_{o}\right) T}+b_{2} \mathbf{f}_{2}^{+\left(k_{o}\right) T}+b_{3} \mathbf{f}_{3}^{+\left(k_{o}\right) T} \\
\mathbf{f}_{1}^{+\left(k_{o}\right) T}+\Delta k \chi \mathbf{f}_{2}^{+\left(k_{o}\right) T}+(\Delta k \chi)^{2} \mathbf{f}_{3}^{+\left(k_{o}\right) T} \\
\mathbf{f}_{1}^{+\left(k_{o}\right) T}+\Delta k \chi^{2} \mathbf{f}_{2}^{+\left(k_{o}\right) T}+\left(\Delta k \chi^{2}\right)^{2} \mathbf{f}_{3}^{+\left(k_{o}\right) T}
\end{array}\right)^{T} \\
& +O\left(\Delta k^{3}\right) .
\end{aligned}
$$

The determinant of $M_{+}^{(\text {prop) }}$ is calculated in full to the lowest order in $\Delta k$ in Appendix as

$$
\operatorname{det}\left(M_{+}^{(\text {prop })}\right)=b_{3} \operatorname{det}\left(S_{+}\right)\left(\chi^{2}-\chi\right) \Delta k \propto \Delta k .
$$

This illustrates that to lowest order this only depends on $b_{3}$, which is the dependence of the input field on $\mathbf{f}_{3}^{+\left(k_{o}\right)}$. Using Eqs. (35) and (38) we find

$$
\begin{aligned}
a_{\text {prop }} & =\frac{\operatorname{det}\left(M^{(\text {prop })_{+}}\right)}{\operatorname{det}\left(M_{+}\right)} \\
& =\frac{b_{3} \operatorname{det}\left(S_{+}\right)\left(\chi^{2}-\chi\right) \Delta k}{\operatorname{det}\left(S_{+}\right) \Delta k^{3} \operatorname{det}\left(M_{V M}\right)} \propto \frac{\Delta k}{\Delta k^{3}}=\Delta k^{-2} .
\end{aligned}
$$

This illustrates the general rule, according to which the denominator in Cramer's rule is proportional to $M_{+} \propto \Delta k^{m(m-1) / 2}$ if all modes are excited, whereas the numerator $M_{+}^{(\text {prop })} \propto$ $\Delta k^{m(m-1) / 2-(m-1)}$. This can be proven for each even $m$ by using the same arguments.

We now confirm these results by numerical calculating the coupling efficiency $\eta$ for different values of $m$. First, the condition on $\delta_{j}$ for a DBE was found for a given set of group velocities $v_{j}$ and coupling coefficients $\rho_{j}$. For a DBE of order $m$, a system with $m / 2=s$ modes was chosen as it is the smallest system that supports it (see Sec. II C). We consider a DBE on the upper side of the band gap, without loss of generality. The exact set of eigenmodes and eigenvalues was calculated directly from the operator $\Theta$ for frequencies close to the DBE. The complex dispersion is plotted from the calculated eigenvalues in Figs. 4(a), 4(c), 4(e), and 4(f) for quadratic, quartic, sextic, and octic DBEs, respectively.

Following Eq. (26), the minimum reflectivity, i.e., the highest coupling, was found for an incident field satisfying (27) and thus carrying unit power. In Fig. 6(a) we show the result of the numerically calculated coupling efficiency $\eta$ versus the group velocity $v_{g}$, where $v_{g}=\partial \omega / \partial k$. Figure 6(a) shows that the higher the order of the DBE, the higher the coupling efficiency for small group velocities. Figure 6(b) that shows the same information on a logarithmic scale. These results are (a)

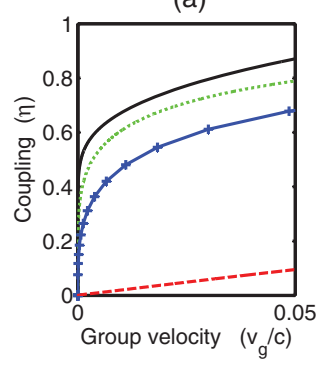

(b)

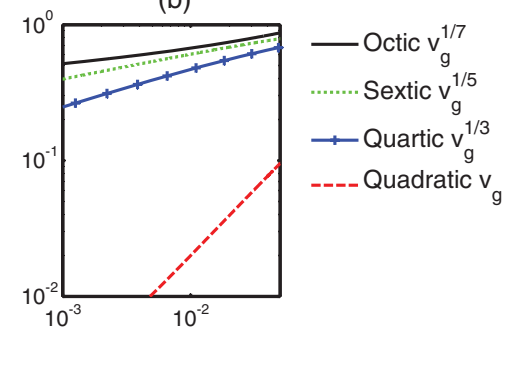

FIG. 6. (Color online) Maximum coupling efficiency versus group velocity close to DBEs of different orders: (a) linear scale and (b) logarithmic scale calculated numerically. All DBEs are for $k_{o}=0$ and $\delta_{1}=0$. Quadratic: dashed red, $v_{1}=1, \rho_{1}=1$. Quartic: blue (plus symbol), $v_{1}=1, v_{2}=0.95, \rho_{1}=, \rho_{2}=0.5$, and $\delta_{2}=-1.765$. Sextic: dotted green, $v_{1}=1, v_{2}=0.95, v_{3}=0.92$, $\rho_{1}=1, \rho_{2}=0.57, \rho_{3}=0.67, \delta_{2}=0.03731$, and $\delta_{3}=1.991$. Octic: black, $v_{1}=1, v_{2}=0.96, v_{3}=0.92, v_{4}=0.9, \rho_{1}=1, \rho_{2}=0.57$, $\rho_{3}=0.67, \rho_{4}=0.4, \delta_{2}=0.1222, \delta_{3}=2.178$, and $\delta_{4}=0.9964$. 
consistent with analytic Eq. (30), confirming that the scaling of the maximum coupling follows $v_{g}^{1 /(m-1)}$.

\section{Coupling to some evanescent modes}

When only some of the evanescent modes are excited with the propagating mode, an additional condition exists on the input field. Continuing with the sextic case, in order to excite only one evanescent mode, a condition on the third input channel $i_{3}$ is given. This can be represented as

$$
\begin{aligned}
\left(\begin{array}{c}
i_{1} \\
i_{2} \\
0
\end{array}\right)= & \left(\begin{array}{c}
\mathbf{f}_{1}^{+\left(k_{o}\right) T}+\Delta k \mathbf{f}_{2}^{+\left(k_{o}\right) T}+\Delta k \mathbf{f}_{3}^{+\left(k_{o}\right) T} \\
\mathbf{f}_{1}^{+\left(k_{o}\right) T}+\Delta k \chi \mathbf{f}_{2}^{+\left(k_{o}\right) T}+(\Delta k \chi)^{2} \mathbf{f}_{3}^{+\left(k_{o}\right) T} \\
c_{1} \mathbf{f}_{1}^{+\left(k_{o}\right) T}+c_{2} \mathbf{f}_{2}^{+\left(k_{o}\right) T}+c_{3} \mathbf{f}_{3}^{+\left(k_{o}\right) T}
\end{array}\right)^{T} \\
& \times\left(\begin{array}{c}
a_{1} \\
a_{2} \\
i_{3}
\end{array}\right)+O\left(\Delta k^{3}\right)
\end{aligned}
$$

in which we express $\left[\begin{array}{lll}0 & 0 & -1\end{array}\right]^{T}$ as a linear combination $c_{1} \mathbf{f}_{1}^{+\left(k_{o}\right)}+c_{2} \mathbf{f}_{2}^{+\left(k_{o}\right)}+c_{3} \mathbf{f}_{3}^{+\left(k_{o}\right)}$. Following the procedure in Appendix, the determinant of the matrix in Eq. (40) is found to the lowest order of $\Delta k$. By replacing the first column by the other input fields $\left[\begin{array}{lll}i_{1} & i_{2} & 0\end{array}\right]^{T}$ expressed as a linear combination of $\left[b_{1} \mathbf{f}_{1}^{+\left(k_{o}\right)}+b_{2} \mathbf{f}_{2}^{+\left(k_{o}\right)}+b_{3} \mathbf{f}_{3}^{+\left(k_{o}\right)}\right]$ and calculating the determinant of it, we find the scaling of the amplitude of the propagating mode:

$$
a_{\text {prop }}=\frac{-\operatorname{det}\left(S_{+}\right)\left(b_{2} c_{3}-b_{3} c_{2}\right)}{b_{3} \operatorname{det}\left(S_{+}\right) \Delta k} \propto \Delta k^{-1} \text {. }
$$

When exciting only the propagating mode, with no evanescent, we also impose a condition on $i_{2}$ by replacing the second column with $\left[d_{1} \mathbf{f}_{1}^{+\left(k_{o}\right) T}+d_{2} \mathbf{f}_{2}^{+\left(k_{o}\right) T}+d_{3} \mathbf{f}_{3}^{+\left(k_{o}\right) T}\right]=$ $\left[\begin{array}{lll}0 & -1 & 0\end{array}\right]$. We find

$$
a_{\text {prop }}=\frac{\operatorname{det}\left(S_{+}\right)\left(\operatorname{det}\left[\begin{array}{lll}
V_{b} & V_{d} & V_{c}
\end{array}\right]\right)}{\operatorname{det}\left(S_{+}\right)\left(d_{2} c_{3}-d_{3} c_{2}\right)} \propto 1,
$$

where $V_{b}, V_{d}$, and $V_{c}$ are the vectors containing the coefficient $b_{j}, d_{j}$, and $c_{j}$, respectively.

Equations (41) and (42) show how the amplitude of the propagating mode scales with the wave vector close to even SPs when one or two evanescent modes are excited. The results are consistent with Eq. (29).

For the cases considered in this section the matrices in Eqs. (25a) and (25b) are not square. A singular value decomposition (SVD) of $M_{+}$was used to find the reflection matrix $R$. For each possible number of evanescent modes excited, the highest coupling efficiency was found. Figure 7(a) shows the coupling efficiency versus group velocity close to a sextic DBE. When only evanescent modes are excited the coupling must be zero (dotted black curve). When exciting only the propagating mode the coupling depends linearly on the group velocity (red,grey curve), as in the quadratic case (red dashed curve in Fig. 6). When evanescent modes are excited with the propagating mode the coupling is enhanced. For comparison with the analytical results of Eq. (30), Fig. 7(b) shows the same results but on a logarithmic scale. (a)

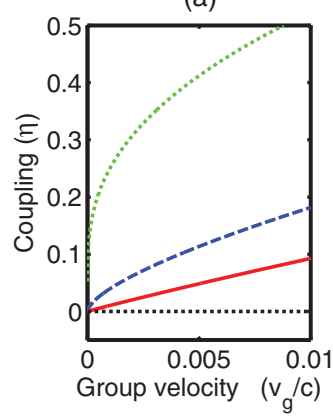

(b)

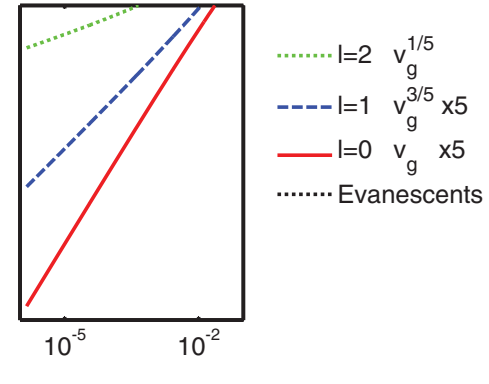

FIG. 7. (Color online) Coupling efficiency versus group velocity for different sets of modes close to a sextic DBE. The parameters are given in the caption to Fig. 6: (a) linear scale and (b) logarithmic scale. Dotted green: propagating mode and both evanescent modes. Dashed blue: propagating and one evanescent mode. Red (gray): only propagating modes. Black: only evanescent modes.

\section{B. Odd stationary points}

At SIPs and DBEs with $k_{o} \neq 0$, the matrix $M_{+}$represents modes that are not all degenerate at the SP frequency. For example, close to a cubic SIP in a three-mode system, there is one mode in the matrix $M_{+}$which is not degenerate with $+k_{o}$ as it has a wave number similar to $-k_{o}$. Nevertheless, when calculating the boundary condition the matrix $M_{+}$must contain every physically possible forward mode

$$
\begin{aligned}
M_{+}= & \left(\begin{array}{c}
\mathbf{f}_{1}^{+\left(k_{o}\right) T}+\Delta k \mathbf{f}_{2}^{+\left(k_{o}\right) T}+\Delta k^{2} \mathbf{f}_{3}^{+\left(k_{o}\right) T} \\
\mathbf{f}_{1}^{+\left(k_{o}\right) T}+\Delta k \chi \mathbf{f}_{2}^{+\left(k_{o}\right) T}+(\Delta k \chi)^{2} \mathbf{f}_{3}^{+\left(k_{o}\right) T} \\
\mathbf{f}_{1}^{+\left(-k_{o}\right) T}+\Delta k \chi^{2} \mathbf{f}_{2}^{+\left(-k_{o}\right) T}+\Delta k \chi^{4} \mathbf{f}_{3}^{+\left(-k_{o}\right) T}
\end{array}\right)^{T} \\
& +O\left(\Delta k^{3}\right),
\end{aligned}
$$

where $\chi=e^{i 2 \pi / 3}$.

Since $\mathbf{f}_{1}^{+\left(-k_{o}\right)}$ is not linearly dependent on $\mathbf{f}_{1}^{+\left(k_{o}\right)}$ and $\mathbf{f}_{2}^{+\left(k_{o}\right)}$, this determinant to the lowest order in $\Delta k$ is

$$
\operatorname{det}\left(M_{+}\right)=\Delta k(1-\chi) \operatorname{det}\left(S_{+}\right),
$$

where $S_{+}=\left[\begin{array}{lll}\mathbf{f}_{1}^{+\left(k_{o}\right)} & \mathbf{f}_{2}^{+\left(k_{o}\right)} & \mathbf{f}_{1}^{+\left(-k_{o}\right)}\end{array}\right]$. The denominator in Cramer's rule is $\operatorname{det}\left(M_{+}^{\text {(prop) }}\right)=b_{2} \operatorname{det}\left(S_{+}\right)$, and we find the amplitude of the propagating mode close to an SIP is

$$
a_{\text {prop }}=\frac{\operatorname{det}\left(M_{+}^{(\mathrm{prop})}\right)}{\operatorname{det}\left(M_{+}\right)}=\frac{b_{2} \operatorname{det}\left(S_{+}\right)}{\Delta k(1-\chi) \operatorname{det}\left(S_{+}\right)}=\frac{b_{2}}{\Delta k(1-\chi)} \text {. }
$$

This occurs when the single degenerate evanescent mode of the cubic SIP is excited, in agreement with Eq. (29) for $l=1$. In higher-ordered SIPs, a larger number of degenerate evanescent modes exist, i.e., two for quintic $\left(k^{5}\right)$ and four for septic $\left(k^{7}\right)$. Using the process shown here, for all the degenerate evanescent modes, and in Sec. III A 2 for part of them, Eq. (29) can be shown for each case.

The difference between SIPs and DBEs is that although in both cases the amplitudes of the modes diverges the same $\left(1 / \Delta k^{l}\right)$, in SIPs the group velocity vanishes slower, which serves to couple to modes with zero group velocity-hence the term "frozen light" [24]. In the cubic case,

$$
\eta \propto\left|a_{\text {prop }}\right|^{2} v_{g} \propto\left(\Delta k^{-1}\right)^{2} \Delta k^{2} \propto 1 .
$$


(a)

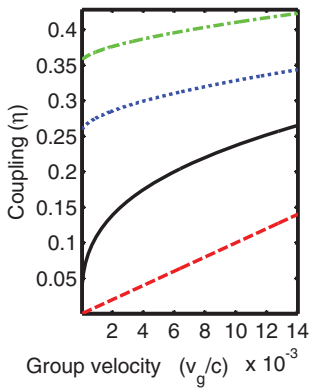

(b)

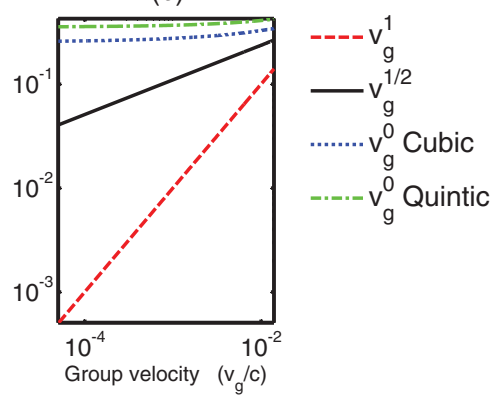

FIG. 8. (Color online) Slow light coupling efficiency close to cubic and quintic SIPs versus group velocity: (a) linear scale and (b) logarithmic scale. Dash red: coupling only to the propagating mode. Black: coupling to the propagating and a single evanescent mode in the quintic case. Dotted blue: coupling to both propagating and the single evanescent mode in the cubic case. Dash-dot green: coupling to the propagating and the two evanescent modes in the quintic case. Cubic: $v_{1}=1, v_{2}=0.95, v_{3}=0.92, \rho_{1}=1, \rho_{2}=0.57$, $\rho_{3}=0.67, \delta_{2}=1.488, \delta_{3}=2.214$, and $k_{o}=0.7$. Quintic: $v_{1}=1$, $v_{2}=0.95, v_{3}=0.93, v_{4}=0.82, v_{5}=0.77, \rho_{1}=1, \rho_{2}=0.35, \rho_{3}=$ $0.8, \rho_{4}=0.8, \rho_{5}=0.31, \delta_{2}=-0.8689, \delta_{3}=0.2101, \delta_{4}=-2.817$, $\delta_{5}=-1.227$, and $k_{o}=0.7$.

As for the DBEs, we now confirm these results by numerical calculating the coupling efficiency, $\eta$ for $m=3$ and $m=5$. First the $\delta_{j}$ 's which lead to cubic $\left(k^{3}\right)$ and quintic $\left(k^{5}\right)$ SIPs were found. Their values are given in the caption to Fig. 8. The center wave number is required to be $k_{o} \neq 0$, as discussed in Sec. II C. Figure 8 shows the numerically calculated coupling efficiency close to and at the cubic and quintic SIPs. When all evanescent modes are excited (one for the cubic SIP, two for quintic SIP), the coupling remains finite at the SIP frequency in contrast to the behavior at even SIPs. When fewer evanescent modes are excited, the efficiency vanishes when $v_{g} \rightarrow 0$, consistent with Eq. (30).

\section{Imperfect stationary points}

In cases where the conditions (21) for an SP are not exactly fulfilled, the modes are not completely degenerate at the SP point. The complex dispersion maintains their structure except very close to the SP where the dispersion lines do not all connect. Figure 9(b) shows the complex dispersion close to
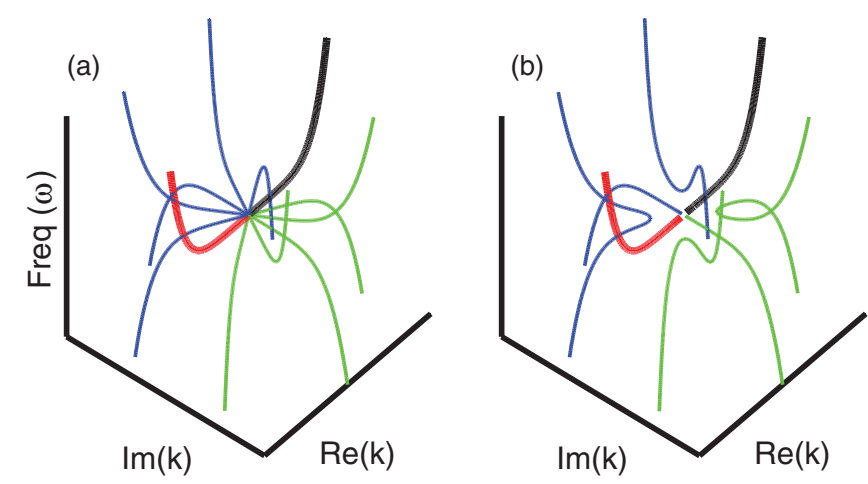

FIG. 9. (Color online) Complex dispersions of (a) perfect and (b) imperfect sextic DBEs. an imperfect sextic DBE for which only some of the modes are perfectly degenerate. Further from the degeneracy point the complex dispersion and as such the spatial structure of the grating modes are not largely effected. This similarity enables the large amplitudes of the propagating modes, and hence the large coupling. At an imperfect SP the amplitude is large but does not diverge.

\section{DISCUSSION AND CONCLUSIONS}

We have shown that evanescent modes can be very important when considering the field at the boundary between two different waveguides. This is the reason why a simple argument, based on the mismatch of impedance, which suggests that the coupling efficiency into a slow mode is necessarily poor, is incorrect. The mechanism is illustrated in Fig. 2. The evanescent modes, which must be present near DBEs and SIPs, help match the slowly propagating mode to a fast mode across the interface. Both propagating and evanescent modes have large amplitudes, which diverge as the DBE or SIP is approached, but interfere destructively at the interface so the total field strength is low, allowing coupling to the fast mode. Away from the interface, the evanescent modes decay away and only the propagating mode is left. The energy carried by the propagating mode is not only related to its amplitude, but also to its group velocity. As the frequency is varied so that an SP is approached, the group velocity decreases, reducing the energy flow. The advantage of DBEs and SIPs is the increased amplitude and hence an enhanced energy flow.

The difference between SPs, and specially between SIPs and DBEs, is the interplay between the diverging amplitude of the propagating mode and the decreasing group velocity. In Table I we summarize these for each SP, where the total energy flow and the coupling efficiency are given. Note in particular that cubic and quartic points ( $m=3,4$, respectively) have the same number of evanescent modes, but that the different

TABLE I. Summary of the coupling efficiency for odd and even SPs, of order $m$ versus the number of excited modes evanescent $l$. Column $s$ gives the number of modes in the homogeneous waveguide which is needed to generate the SP; column $v_{g}$ gives the scaling of the group velocity with the wave number $\Delta k ; a_{\text {prop }}$ gives the scaling of the amplitude of the propagating modes; and $\eta$ the scaling of the coupling efficiency with group velocity.

\begin{tabular}{lcccccc}
\hline \hline SP & $m$ & $s$ & 1 & $v_{g}$ & $a_{\text {prop }}$ & $\eta$ \\
\hline Quadratic & 2 & 1 & 0 & $\Delta k$ & 1 & $v_{g}$ \\
Cubic & 3 & 3 & 0 & $\Delta k^{2}$ & 1 & $v_{g}$ \\
& & & 1 & & $\Delta k^{-1}$ & 1 \\
Quartic & 4 & 2 & 0 & $\Delta k^{3}$ & 1 & $v_{g}$ \\
& & & 1 & $\Delta k^{3}$ & $\Delta k^{-1}$ & $v_{g}^{1 / 3}$ \\
Quintic & 5 & 5 & 0 & $\Delta k^{4}$ & 1 & $v_{g}$ \\
& & & 1 & & $\Delta k^{-1}$ & $v_{g}^{2 / 4}$ \\
& & & 2 & & $\Delta k^{-2}$ & 1 \\
Sextic & 6 & 3 & 0 & $\Delta k^{5}$ & 1 & $v_{g}$ \\
& & & 1 & & $\Delta k^{-1}$ & $v_{g}^{3 / 5}$ \\
& & & 2 & & $\Delta k^{-2}$ & $v_{g}^{1 / 5}$ \\
\hline \hline
\end{tabular}


behavior stems from the different group velocities. Similar arguments apply to quintic and sextic points, etc.

A key finding is that for SIPs the coupling efficiency does not vanish as $v_{g} \rightarrow 0$, corresponding to frozen light [24]. This is only so if all degenerate modes are coupled: one for cubic SIPs, two for quintic SIPs, etc. On the other hand, at DBEs the amplitude of the propagating mode diverges but the group velocity decreases faster. Nonetheless, even though true frozen light only occurs at SIPs for which all evanescent modes are excited, the coupling efficiency for DBEs can be significantly larger than for quadratic stationary points. Therefore the use of DBEs may still be preferable since the required complexity of the structure is lower, i.e., the number of modes $s=2$ for a quartic DBE versus an $s=3$ waveguide for a cubic SIP (see Table I). The difference occurs because DBEs can be generated at the BZ center or edge, whereas SIPs can only occur in pairs at $\pm k_{o}$. The generic incident field to a waveguide, featuring an SP, will excite the maximum number of degenerate evanescent modes $l$, as it is necessary to have a specific field profile to prevent a mode from being excited. We emphasize that although different decaying evanescent modes can be excited at the interface, the number $l$ stand for modes that are degenerate with the propagating mode, for example, associated with $k_{o}$ at the SP (corresponding to the same Jordan block, Sec. III A 2).

As mentioned in Sec. I, one of the aims in exploring the coupled wave-guiding system in this paper is to help understand the more complicated PC-based structures with large refractive index contrasts [31]. Although it is hard to treat such geometries analytically, numerical [22,32,33] and experimental [15] results agree with our main findings. This includes the number of modes required to generate a particular dispersion relation feature (parameter $s$ in Table I), as well as the scaling of the efficiency with group velocity when the maximum number of degenerate evanescent modes $l$ is excited.
We surmise that the scaling of the efficiency for other values of $s, l$, and $m$ also carries over to high-index materials.

\section{ACKNOWLEDGMENTS}

This work was supported by the Australian Research Council. The authors thank Ross McPhedran, Chris Poulton, and Kokou Dossou for fruitful discussions

\section{APPENDIX: THE DETERMINANTS OF $M_{+}$AND $M_{+}^{(\text {prop })}$}

We present an example of how the dependence on $\Delta k$, to the lowest order, was found for the denominator $M_{+}^{(\text {prop })}$ and the numerator $M_{+}$in Cramer's rule. The case of the sextic DBE when all the evanescent modes are excited is given.

The determinant of $M_{+}$is easily found as it is a multiplication of three matrices [Eq. (33)]. We calculate $M_{+}^{(\mathrm{prop})}$, which is $M_{+}$when replacing the first column by the incident field, also given as Eq. (37):

$$
\begin{aligned}
M_{+}^{(\text {prop })}= & \left(\begin{array}{c}
b_{1} \mathbf{f}_{1}^{+\left(k_{o}\right) T}+b_{2} \mathbf{f}_{2}^{+\left(k_{o}\right) T}+b_{3} \mathbf{f}_{3}^{+\left(k_{o}\right) T} \\
\mathbf{f}_{1}^{+\left(k_{o}\right) T}+\Delta k \chi \mathbf{f}_{2}^{+\left(k_{o}\right) T}+(\Delta k \chi)^{2} \mathbf{f}_{3}^{+\left(k_{o}\right) T} \\
\mathbf{f}_{1}^{+\left(k_{o}\right) T}+\Delta k \chi^{2} \mathbf{f}_{2}^{+\left(k_{o}\right) T}+\left(\Delta k \chi^{2}\right)^{2} \mathbf{f}_{3}^{+\left(k_{o}\right) T}
\end{array}\right)^{T} \\
& +O\left(\Delta k^{3}\right) .
\end{aligned}
$$

The determinant does not change when permutating the rows:

$$
\operatorname{det}\left(M_{+}^{\mathrm{prop}}\right)=\left|\begin{array}{c}
\mathbf{f}_{1}^{+\left(k_{o}\right) T}+\Delta k \chi \mathbf{f}_{2}^{+\left(k_{o}\right) T}+(\Delta k \chi)^{2} \mathbf{f}_{3}^{+\left(k_{o}\right) T} \\
\mathbf{f}_{1}^{+\left(k_{o}\right) T}+\Delta k \chi^{2} \mathbf{f}_{2}^{+\left(k_{o}\right) T}+\left(\Delta k \chi^{2}\right)^{2} \mathbf{f}_{3}^{+\left(k_{o}\right) T} \\
b_{1} \mathbf{f}_{1}^{+\left(k_{o}\right) T}+b_{2} \mathbf{f}_{2}^{+\left(k_{o}\right) T}+b_{3} \mathbf{f}_{3}^{+\left(k_{o}\right) T}
\end{array}\right| .
$$

Next, we eliminate the dependence of the second and third rows on $f_{1}$ and factorize $k\left(\chi^{2}-\chi\right)$ from the second row, followed by eliminating $f_{2}$ from the third row:

$$
\begin{aligned}
\operatorname{det}\left(M_{+}^{\text {prop }}\right) & =\left|\begin{array}{c}
\mathbf{f}_{1}^{+\left(k_{o}\right) T}+\Delta k \chi \mathbf{f}_{2}^{+\left(k_{o}\right) T}+(\Delta k \chi)^{2} \mathbf{f}_{3}^{+\left(k_{o}\right) T} \\
\Delta k\left(\chi^{2}-\chi\right) \mathbf{f}_{2}^{+\left(k_{o}\right) T}+\Delta k^{2}\left(\chi^{4}-\chi^{2}\right) \mathbf{f}_{3}^{+\left(k_{o}\right) T} \\
\left(b_{2}-b_{1} \Delta k \chi\right) \mathbf{f}_{2}^{+\left(k_{o}\right) T}+\left[b_{3}-b_{1}(\Delta k \chi)^{2}\right] \mathbf{f}_{3}^{+\left(k_{o}\right) T}
\end{array}\right| \\
& =\Delta k\left(\chi^{2}-\chi\right)\left|\begin{array}{c}
\mathbf{f}_{1}^{+\left(k_{o}\right) T}+\Delta k \chi \mathbf{f}_{2}^{+\left(k_{o}\right) T}+(\Delta k \chi)^{2} \mathbf{f}_{3}^{+\left(k_{o}\right) T} \\
\mathbf{f}_{2}^{+\left(k_{o}\right) T}+\Delta k\left(\chi^{2}+\chi\right) \mathbf{f}_{3}^{+\left(k_{o}\right) T} \\
\left(b_{2}-b_{1} \Delta k \chi\right) \mathbf{f}_{2}^{+\left(k_{o}\right) T}+\left[b_{3}-b_{1}(\Delta k \chi)^{2}\right] \mathbf{f}_{3}^{+\left(k_{o}\right) T}
\end{array}\right| \\
& =\Delta k\left(\chi^{2}-\chi\right)\left|\begin{array}{c}
\mathbf{f}_{1}^{+\left(k_{o}\right) T}+\Delta k \chi \mathbf{f}_{2}^{+\left(k_{o}\right) T}+(\Delta k \chi)^{2} \mathbf{f}_{3}^{+\left(k_{o}\right) T} \\
\mathbf{f}_{2}^{+\left(k_{o}\right) T}+\Delta k\left(\chi^{2}-\chi\right) \mathbf{f}_{3}^{+\left(k_{o}\right) T} \\
{\left[\left[b_{3}-b_{1}(\Delta k \chi)^{2}\right]-\left[b_{2}-b_{1}(\Delta k \chi)\right] \Delta k\left(\chi^{2}+\chi\right)\right\} \mathbf{f}_{3}^{+\left(k_{o}\right) T}}
\end{array}\right| .
\end{aligned}
$$

Taking the lowest order of the matrix determinant,

$$
\operatorname{det}\left(M_{+}^{(\text {prop })}\right)=b_{3} \Delta k\left(\chi^{2}-\chi\right) \operatorname{det}\left(\mathbf{f}^{+\left(k_{o}\right)} \quad \mathbf{f}_{2}^{+\left(k_{o}\right)} \quad \mathbf{f}_{3}^{+\left(k_{o}\right)}\right)=b_{3} \Delta k\left(\chi^{2}-\chi\right) \operatorname{det}\left(S_{+}\right)+O\left(\Delta k^{2}\right) .
$$

This shows that, to the lowest order, the determinant of $M^{\text {prop }} \propto \Delta k$ and it is only dependent on $c_{3}$ of the incident field. 
[1] T. Baba, Nat. Photonics 2, 465 (2008).

[2] A. Figotin and I. Vitebsky, Phys. Rev. E 63, 066609 (2001).

[3] A. A. Sukhorukov, C. J. Handmer, C. M. de Sterke, and M. J. Steel, Opt. Express 15, 17954 (2007).

[4] J. B. Khurgin and J. B. Tucker, Slow Light: Science and Applications, Vol. 140 (CRC Press, Boca Raton, FL, 2008).

[5] R. W. Boyd, J. Opt. Soc. Am. B 28, A38 (2011).

[6] N. A. Mortensen and S. Xiao, Appl. Phys. Lett. 90, 141108 (2007).

[7] N. Gutman, A. Armon, A. Saar, A. Osherov, and Y. Golan, Appl. Phys. Lett. 93, 073111 (2008).

[8] C. Monat, B. Corcoran, M. Ebnali-Heidari, C. Grillet, B. J. Eggleton, T. P. White, L. O'Faolain, and T. F. Krauss, Opt. Express 17, 2944 (2009).

[9] C. Monat, M. Ebnali-Heidari, C. Grillet, B. Corcoran, B. Eggleton, T. White, L. O'Faolain, J. Li, and T. Krauss, Opt. Express 18, 22915 (2010).

[10] L. Van Hove, Phys. Rev. 95, 249 (1954).

[11] K. Sakoda, Opt. Express 4, 167 (1999).

[12] J. Ballato, A. Ballato, A. Figotin, and I. Vitebskiy, Phys. Rev. E 71, 036612 (2005).

[13] A. Figotin and I. Vitebskiy, Phys. Rev. E 68, 036609 (2003).

[14] Y. A. Vlasov, M. O’Boyle, H. F. Hamann, and S. J. McNab, Nature (London) 438, 65 (2005).

[15] M. Spasenović, T. P. White, S. Ha, A. A. Sukhorukov, T. Kampfrath, Y. S. Kivshar, C. M. de Sterke, T. F. Krauss, and L. Kuipers, Opt. Lett. 36, 1170 (2011).

[16] A. A. Sukhorukov, S. Ha, A. S. Desyatnikov, A. V. Lavrinenko, and Y. S. Kivshar, J. Opt. A 11, 094016 (2009).

[17] S. Ha, A. A. Sukhorukov, A. V. Lavrinenko, and Y. S. Kivshar, Photonics Nanostruct. Fundam. Appl. 8, 310 (2010).
[18] A. Figotin and I. Vitebskiy, J. Magn. Magn. Mater. 300, 117 (2006).

[19] A. Figotin and I. Vitebskiy, Phys. Rev. E 74, 066613 (2006).

[20] N. Gutman, L. C. Botten, A. A. Sukhorukov, and C. M. de Sterke, Opt. Lett. 36, 3257 (2011).

[21] T. Tamir, Integrated Optics, Topics in Applied Physics (Springer, Berlin, 1979), Vol. 7.

[22] T. P. White, L. C. Botten, C. M. de Sterke, K. B. Dossou, and R. C. McPhedran, Opt. Lett. 33, 2644 (2008).

[23] S. Ha, M. Spasenović, A. A. Sukhorukov, T. P. White, C. M. de Sterke, K. L. Kuipers, T. F. Krauss, and Y. S. Kivshar, J. Opt. Soc. Am. B 28, 955 (2011).

[24] A. Figotin and I. Vitebskiy, Waves Random Complex Media 16, 293 (2006).

[25] B. S. Kawasaki, K. O. Hill, D. C. Johnson, and Y. Fujii, Opt. Lett. 3, 66 (1978).

[26] G. Meltz, W. W. Morey, and W. H. Glenn, Opt. Lett. 14, 823 (1989).

[27] V. Lidskii, U.S.S.R. Comput. Math. Math. Phys. 1, 73 (1965).

[28] J. Moro, J. V. Burke, and M. L. Overton, SIAM J. Matrix Anal. Appl. 18, 793 (1997).

[29] B. Noble and J. Daniel, Applied Linear Algebra, 2nd ed. (Prentice-Hall, Englewood Cliffs, NJ, 1977).

[30] L. C. Botten, T. P. White, A. A. Asatryan, T. N. Langtry, C. Martijn de Sterke, and R. C. McPhedran, Phys. Rev. E 70, 056606 (2004).

[31] H. Kurt, H. Benisty, T. Melo, O. Khayam, and C. Cambournac, J. Opt. Soc. Am. B 25, C1 (2008).

[32] C. M. de Sterke, K. B. Dossou, T. P. White, L. C. Botten, and R. C. McPhedran, Opt. Express 17, 17338 (2009).

[33] A. Hosseini, X. Xiaochuan, D. N. Kwong, H. Subbaraman, W. Jiang, and R. T. Chen, Appl. Phys. Lett. 98, 031107 (2011). 